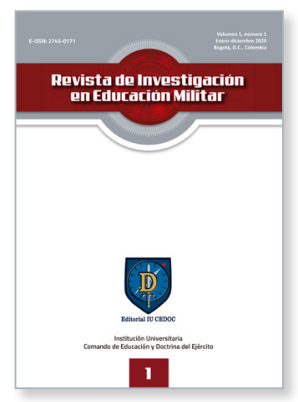

Revista de Investigación en Educación Militar

Volumen 1, número 1, enero-diciembre 2020, pp. 7-9

Bogotá, D. C., Colombia

E-ISSN: 2745-0171

https://doi.org/10.47961/27450171.10

\title{
Editorial: La educación militar como sistema complejo enfocado a formar, capacitar, entrenar y reentrenar personas que brindan seguridad y defensa
}

\section{Jorge M. Cardona-Angarita}

https://orcid.org/0000-0001-7830-1960

jorge.cardonaan@buzonejercito.mil.co

Comando de Educación y Doctrina del Ejército, Bogotá, D. C., Colombia

Citación: Cardona-Angarita, J. M. (2020). Editorial: La educación militar como sistema complejo enfocado a formar, capacitar, entrenar y reentrenar personas que brindan seguridad y defensa. Revista de Investigación en Educación Militar, 1(1), 7-9. DOl: https://doi.org/10.47961/27450171.10

Publicado en línea: $1 .^{\circ}$ de diciembre de 2020

Los artículos publicados por la Revista de Investigación en Educación Militar son de acceso abierto bajo una licencia Creative Commons: Atribución - No Comercial - Sin Derivados.

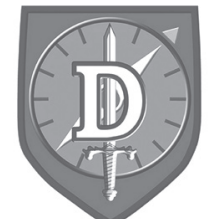

Editorial IU CEDOC 


\section{Editorial: La educación militar como sistema complejo enfocado a formar, capacitar, entrenar y reentrenar personas que brindan seguridad y defensa}

Editorial: Military education as a complex system focused on training and educating people who provide security and defense

Jorge M. Cardona-Angarita

Comando de Educación y Doctrina del Ejército, Bogotá, D. C., Colombia

Durante los tres últimos siglos, la ciencia era la única vía legal para alcanzar la verdad. Su ideal del esquema universal e intemporal, de equilibrio y estabilidad, de la negación de la realidad del tiempo, de que la naturaleza es controlada por leyes deterministas y reversibles, donde es posible predecir o retrodecir cualquier situación pasada o futura del universo, hizo que se produjera la separación entre dos culturas: la de la ciencia y la de la filosofía. Luego, las disposiciones epistemológicas de comienzos del siglo XIX sobre el sujeto como objeto de estudio fueron el resultado de las mutaciones de ese pensamiento clásico y hoy en día no solo acudimos a lógicas clásicas, sino también a las lógicas no clásicas para dar respuesta a problemas sociales ante los nuevos frentes de discursos transdiciplinarios, medioambientales, evolucionistas, cognitivos, biogenéticos y digitales que se están implantando en las sociedades occidentales actuales.

Las ciencias del pasado se definieron por objetos, con disciplinas que tenían un método, un lenguaje, un objeto propio y una tradición específica. Ahora, con diversos enfoques y llamados a la interdisciplinariedad, se habla de una ampliación del campo de frontera con identificación de problemas. En este sentido, la educación militar como rama de las ciencias de la educación está en eclosión y busca a través de las investigaciones científicas comprender una sociedad específica que cumple sus funciones en bien de la humanidad. 
Una sociedad militar puede ser estudiada desde varias perspectivas debido a la influencia que esta ejerce sobre diferentes aspectos, como la economía, la política, la cultura y la seguridad dentro de los Estados. Actualmente, a nivel mundial y en un porcentaje mayor, las instituciones militares son estudiadas por centros educativos, de pensamiento y de estudios estratégicos, en el marco de su eficacia, eficiencia y efectividad dentro del concepto de seguridad, defensa y políticas militares. El estudio de la sociedad militar trasciende esta dimensión y la lleva al campo de la interdisciplinariedad, de manera que aborda aspectos importantes como las relaciones humanas, la cultura, la educación, la formación, la capacitación militar, entre otros.

La educación militar en las instituciones destinadas para ello tiene una consideración o régimen especial, en cuanto es un sistema complejo enfocado a formar, capacitar, entrenar y reentrenar personas para brindar seguridad y defensa. En este sentido y siguiendo a Esquivel Triana (2015), la educación significa el desarrollo personal sobre diferentes grados de conocimiento, de cualidades mentales o de carácter, salud física o competencias específicas para un curso formal o instrucción, mientras que el entrenamiento y el reentrenamiento incluyen la enseñanza, la instrucción y el reforzamiento de ejercicios para desarrollar los potenciales de mente y cuerpo esenciales para proceder en la guerra, tanto de individuos como de unidades tácticas. Asimismo, el sistema de educación de las Fuerzas Militares de Colombia define la formación como un conjunto de áreas del conocimiento que interrelacionan saberes teóricos, prácticos y actitudinales, las cuales crean condiciones en el desarrollo de competencias ya sea de manera básica, investigativa, sociohumanística o profesional. En cuanto a la capacitación, esta busca que el militar adquiera capacidades o habilidades específicas relacionadas con el campo militar con el fin de mejorar los resultados aprendidos en la formación militar.

Consecuente con lo anterior, hoy presentamos la primera edición de la Revista de Investigación en Educación Militar (RIEM), un proyecto editorial y científico que está enfocado a difundir los avances teóricos y prácticos sobre la educación en instituciones que tengan relación con el ámbito militar, con énfasis en la formación, la capacitación, el entrenamiento y el reentrenamiento de hombres y mujeres que conforman la sociedad militar, como también las unidades militares que en conjunto participan en las operaciones militares.

En esta edición se presentan cinco artículos relacionados con la educación, los cuales se convierten en referencia para estudios comparados o como base de futuras investigaciones. El primer artículo, titulado "Las ciencias militares en Colombia y el saber Doctrina Militar, alineación fundamental para la efectividad de las instituciones militares", es una investigación desarrollada por el Comando de Educación y Doctrina (CEDOC) que concluye con una propuesta de plan para fortalecer el saber Doctrina Militar dentro del programa de las ciencias militares; el segundo, "Didáctica de la historia en la educación superior a través de las mediaciones. De la Historia Estelar a la Educación Estelar", hace una revisión documental sobre la enseñanza de la 
historia en Colombia y presenta un estudio de caso en el que se propone una didáctica desde la Historia Estelar con estudiantes de primer semestre de Negocios y Relaciones Internacionales de la Universidad de La Salle; el tercer artículo, "Relaciones entre Colombia y sus vecinos frente a la ayuda internacional", analiza los impactos del Plan Colombia en la región y las consecuencias de la ayuda de Estados Unidos en las negociaciones del Tratado de Libre Comercio y en la soberanía colombiana; el cuarto artículo, "La robótica creativa para el desarrollo de la cultura Maker inclusiva en la educación básica: caso Escuela Municipal de Capistrano de Abreu, en São Paulo, Brasil", es un estudio de caso sobre el aprendizaje de la robótica en una institución de educación básica en São Paulo, Brasil; el quinto y último artículo, titulado "El modelo de tecnología Blockchain en la autenticación de certificados inteligentes para entidades educativas", consiste en la modelación de un sistema Blockchain capaz de implementar el servicio de autenticación de certificados estudiantiles para distintas entidades educativas de la nación.

Estos artículos forman parte de un naciente proyecto sobre la educación militar con enfoque nacional, regional e internacional, que busca la interdisciplinariedad de las diferentes ciencias. Reconocer que la actividad de un grupo es científica, en parte por su semejanza con otros campos del mismo grupo y en parte por su diferencia con otras actividades que pertenecen a otros grupos disciplinarios, es lo que Thomas Kuhn (1989) Ilama la conmensurabilidad y, a su vez, una revolución científica. En este sentido, seguiremos invitando a los investigadores de todas las disciplinas para que amplíen la frontera del conocimiento de la sociedad militar a través de las publicaciones de sus artículos en esta innovadora revista.

\section{Referencias}

Esquivel Triana, R. (2015). Formación e instrucción militar en Colombia, 1880-1906. Boletín de Historia y Antigüedades, 102(860), 85-86.

Kuhn, T. S. (1989). ¿Qué son las revoluciones cientificas? y otros ensayos. Paidós. 